\title{
Solitary Lumbar Spinal Osteochondroma Arising from the L3 Articular Process
}

\author{
Hiroshi Takahashi, ${ }^{1}$ Akihito Wada, ${ }^{1}$ Fumiaki Terajima, ${ }^{1}$ Yuichiro Yokoyama, ${ }^{1}$ Yasuhiro Inoue, ${ }^{1}$ \\ Kazutoshi Shibuya, ${ }^{2}$ Daisuke Sasai, ${ }^{2}$ and Masayuki Sekiguchi ${ }^{1}$ \\ ${ }^{1}$ Department of Orthopaedic Surgery, School of Medicine, Toho University, 6-11-1 Omori-nishi, Ota-ku, Tokyo 143-8541, Japan \\ ${ }^{2}$ Department of Pathology, School of Medicine, Toho University, 5-21-16 Omori-nishi, Ota-ku, Tokyo 143-8540, Japan \\ Address correspondence to Hiroshi Takahashi,drkan@med.toho-u.ac.jp
}

Received 30 September 2012; Accepted 9 October 2012

Copyright (C) 2013 H. Takahashi et al. This is an open access article distributed under the terms of the Creative Commons Attribution License, which permits unrestricted use, distribution, and reproduction in any medium, provided the original work is properly cited.

\begin{abstract}
Solitary lumbar spinal osteochondroma with neurological symptoms is very rare. We report the case of an 81-year-old man with radicular pain caused by lumbar solitary osteochondroma. A computed tomography (CT) scan showed a bony tumor arising from the left L2/3 articular process. Intraoperative findings showed that the tumor originated from the left superior articular process of the L3 vertebra. After surgery, the patient became asymptomatic immediately.
\end{abstract}

Keywords lumbar osteochondroma; lumbar spinal canal; radicular pain

\section{Introduction}

Osteochondroma is one of the more common benign bone tumors and usually occurs in long bones, but is occasionally found in the spine. Spinal osteochondroma more commonly involves the cervical and upper thoracic spine, rather than the lumbar spine [1]. Most of the lesions are asymptomatic because they tend to grow out of the spinal canal [8]. Thus, solitary lumbar spinal osteochondroma with neurological symptoms is rare. Here, we report a case of lumbar osteochondroma causing radicular pain that arose from the left superior articular process of the L3 vertebra.

\section{Case report}

An 81-year-old man presented with complaints of lumbago and thigh pain that had worsened over the preceding 5 months. The patient had undergone conservative treatment given by a local doctor, but this had failed to improve his complaints. Therefore, he was referred to our institute for detailed examination and management. A neurological examination revealed left L2 and L3 hypesthesia. There was no motor weakness. The patient had no history of inherited diseases.

In a plain lumbar Xp lateral view, a faint tumor shadow that was thought to involve bone was observed in the upper inferior border of the L2 vertebra in the spinal canal (Figure 1). A CT scan showed a bony tumor arising from the left
L2/3 articular process. The tumor also involved the cortex and medullary cavity, but continuity with the left L2/3 articular process was unclear. The spinal canal was narrowed by the tumor (Figure 2). The central portion of the tumor gave a signal of increased intensity on MRI and was surrounded by peripheral ossification and a cartilage cap of low signal intensity on T1- and T2-weighted images. MRI also indicated severe compression of the thecal sac at the L2/3 level (Figure 3).

L2 laminectomy and left L2/3 facetectomy were performed for total tumor resection. The tumor was severely

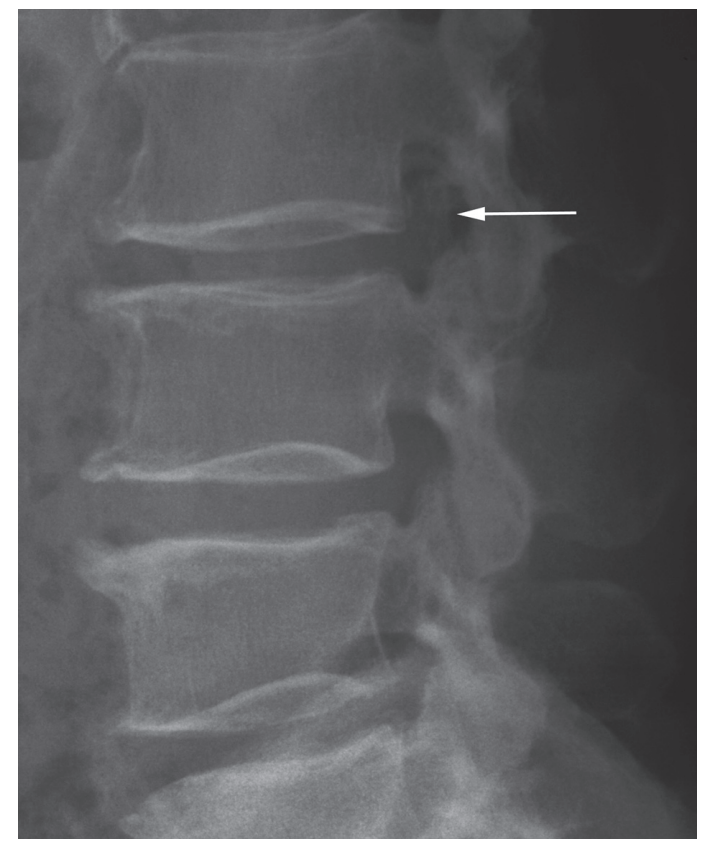

Figure 1: A faint tumor shadow (arrow) that was thought to involve bone was observed in the spinal canal at L2/3 level. 


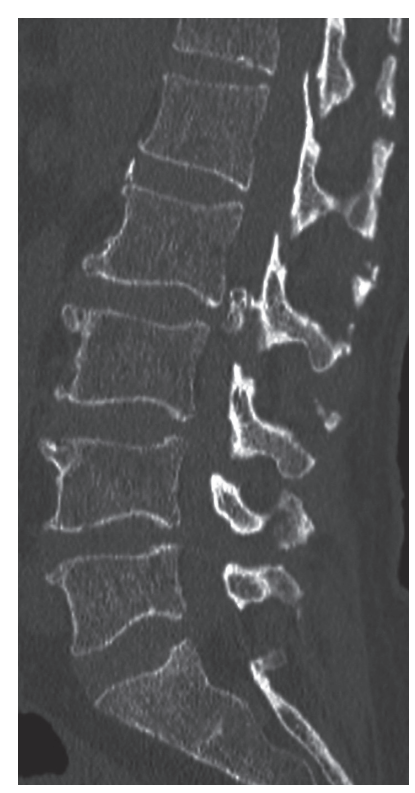

(a)

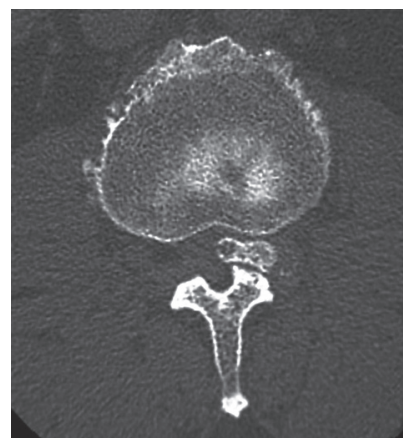

(b)
Figure 2: (a) A bony tumor arising from the left L2/3 articular process. (b) The spinal canal was narrowed by the tumor at $\mathrm{L} 2 / 3$ level.

adhered to the yellow ligament, but the yellow ligament was attached normally to the margin of the articular processes and there was no adhesion between the tumor and the nerve root or dura mater. Intraoperative findings showed that the tumor originated from the left superior articular process of the L3 vertebra. The gross appearance of the resected tumor indicated that it was composed of cartilaginous and osseous components (Figure 4). After en bloc removal of the tumor, interbody fusion was performed using a pedicle screw system.

A histological study confirmed the diagnosis of benign osteochondroma (Figure 5). The patient became asymptomatic immediately after surgery and the postoperative course was uneventful. A radiograph and CT scan at 1 year postoperatively showed complete tumor resection without recurrence.

\section{Discussion}

Osteochondroma is a common benign bone tumor [7], but only $1-4 \%$ of osteochondromas arise in the spine $[1,2,4,6$, 8]. However, spinal osteochondroma may be underreported in relation to the true incidence because most of these tumors usually grow out of the spinal canal, and therefore do not usually cause symptoms $[6,10]$.

Spinal osteochondromas are more commonly found in the cervical and upper thoracic spine and the $\mathrm{C} 1$ or $\mathrm{C} 2$ vertebra is most frequently involved $[2,6,10]$. There are two hypotheses for the preferred development of osteochondroma in the cervical vertebra. Albrecht et al. [1]

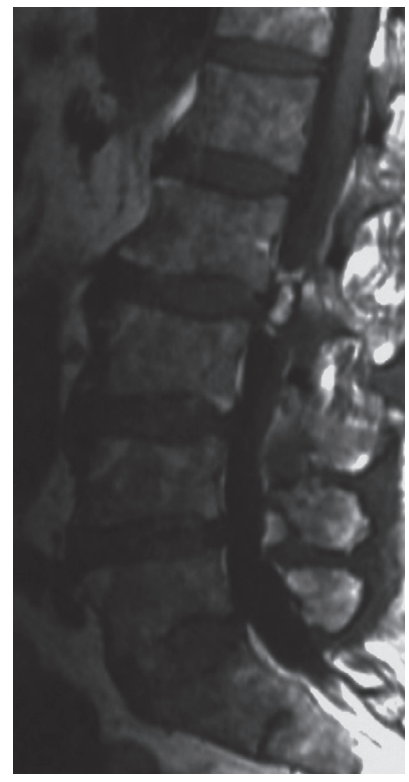

(a)

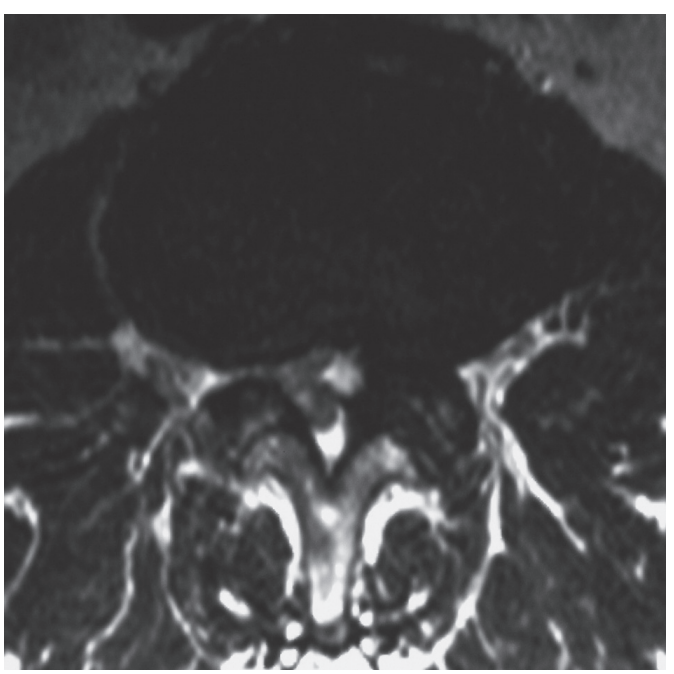

(c)

(b)

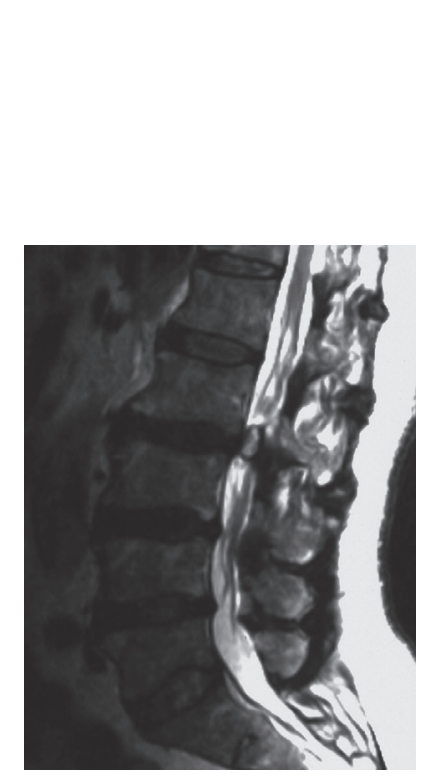
of increased intensity on MRI and was surrounded by peripheral ossification and a cartilage cap of low signal intensity on T1- and T2-weighted images.

suggested that the predominance of cervical lesions is caused by microtrauma inflicted on the epiphyseal cartilage, because of the greater mobility and flexibility of these vertebra. According to this hypothesis, the cervical and lumbar spine should be involved more than the thoracic spine [8]. However, in the cases published in the literature there is clearly a lower incidence of osteochondroma in the lumbar region. In an alternative hypothesis, Fiumara et al. [8] focus on the difference in the time of appearance of secondary ossification centers in the spinous process,
Figure 3: The central portion of the tumor gave a signal 


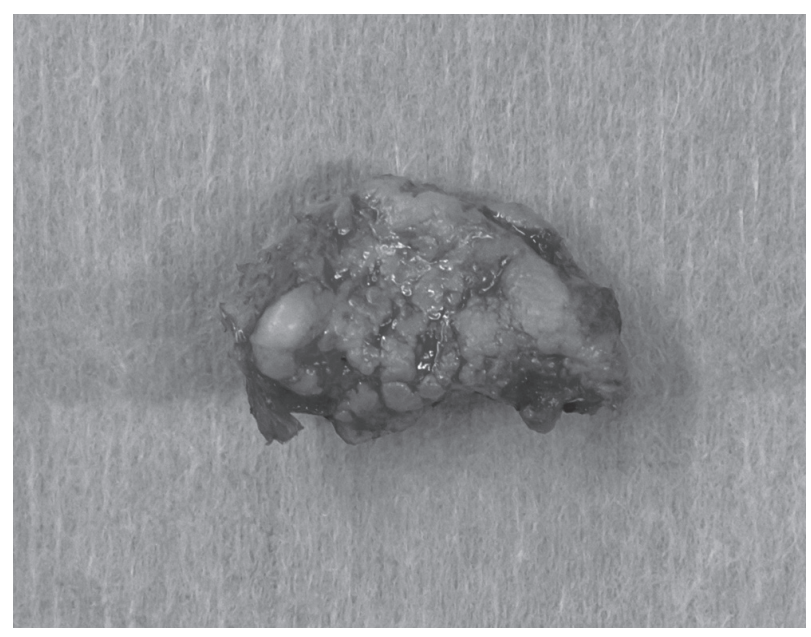

Figure 4: The gross appearance of the resected tumor indicated that it was composed of cartilaginous and osseous components.

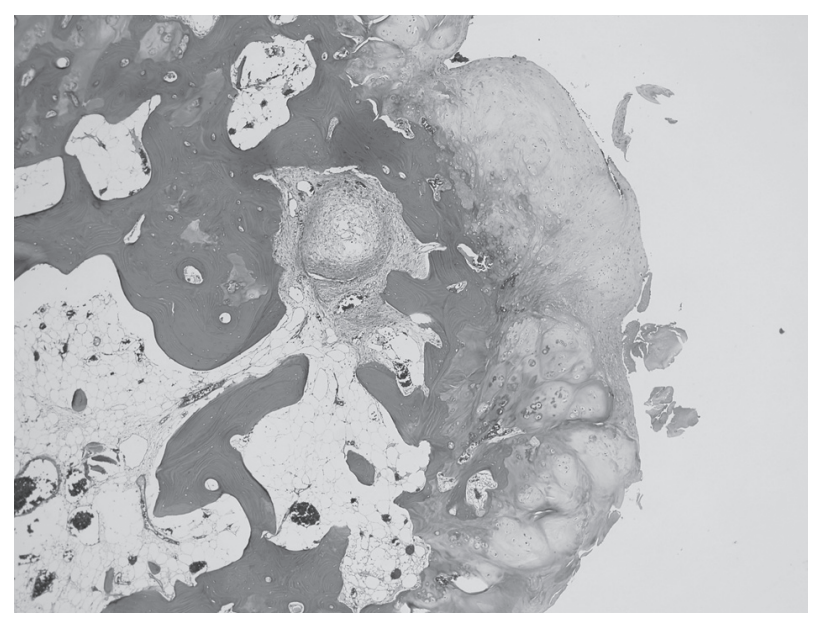

Figure 5: Histological study confirmed the diagnosis of benign osteochondroma.

transverse process, articular process, and endplate of the vertebral body. These centers appear in children between the ages of 11 and 18 years old and develop into complete ossification in the cervical spine during adolescence; in the thoracic and lumbar spines during the end of the second decade of life; and in the sacrum during the third decade of life [16]. Cartilage in these secondary ossification centers may be the origin of aberrant islands of cartilaginous tissue that cause osteochondromas to form. A more rapid ossification process in these centers may be associated with a greater probability that aberrant cartilage will form. Therefore, the more frequent presence of osteochondroma in the upper segments of the vertebral column could be explained by different durations of the ossification processes in the different centers.
Spinal osteochondromas occur in two distinct clinical settings as solitary lesions or in association with multiple hereditary exostosis (MHE). There are conflicting reports on whether solitary osteochondroma or lesions associated with MHE more commonly affect the spine [1,5, 14, 15, 17 , $18,19]$. Findings from the largest clinical series reported by Bess et al. [3] showed that the majority of osteochondromas are solitary rather than associated with MHE. A literature review by Bess et al. showed that the mean age of all patients with spinal osteochondroma was 28.5 years old (range 269 years old) and that the mean age for those with neurological symptoms was 29.7 years old (2-68 years old). Patients with MHE presenting with neurological symptoms were significantly younger than those with solitary lesions and neurological symptoms: 22.3 (range 7-46) versus 36.6 (2-68) years old. Only 10 cases of solitary lumbar spinal osteochondroma with development of sciatica or radicular pain in the lumbar spine have been reported [20], among which the patient in the current case is the oldest.

Our patient developed the disease at a significantly older age than patients in previous reports. However, surgical findings suggested that the tumor had been present for a long period. A detailed disease history did not indicate development of cauda eqina syndrome or radicular pain in the past, and there was no clear trauma during the period when the symptoms were observed. These findings may have been due to the slow development of the tumor. Development of radicular pain may have been caused by minor trauma and gradual narrowing of the spinal canal due to aging.

Recurrence rates after resection of osteochondromas are approximately 2\% [13]. According to Dahlin and Unni [7], the frequency of malignant degeneration is about $1 \%$ in spinal osteochondroma and 10 to $15 \%$ in MHE for all tumor locations. Therefore, complete resection of the lesion is important to prevent recurrence and malignant transformation. Several studies have noted that most osteochondromas show focal exophytic growth in the posterior elements, such as laminae or articular processes, and that total resection of the mass and neural decompression can be achieved without fusion surgery $[8,9,11,12,21]$. However, for some cases with articular process-derived osteochondroma, total facetectomy is required to remove the tumor completely and obtain a negative margin, although this depends on the tumor size. For these cases, spinal fusion is required to prevent postoperative intervertebral instability.

\section{Conclusion}

We experienced a case of solitary spinal osteochondroma with radicular pain in the lumbar vertebra in a patient who was older than those in all past reports of similar cases. Even in elderly patients, this disease should be considered as a candidate in differential diagnosis following imaging of a bony tumor in the spinal canal. 


\section{References}

[1] S. Albrecht, J. S. Crutchfield, and G. K. SeGall, On spinal osteochondromas, J Neurosurg, 77 (1992), 247-252.

[2] H. Atabay, Y. Kuyucu, O. Korkmaz, and A. C. Iplikcioglu, Myelopathy due to hereditary multiple exostoses: CT and MR studies, Clin Neurol Neurosurg, 98 (1996), 186-188.

[3] R. S. Bess, M. R. Robbin, H. H. Bohlman, and G. H. Thompson, Spinal exostoses: analysis of twelve cases and review of the literature, Spine, 30 (2005), 774-780.

[4] G. Borne and C. Payrot, Right lumbo-crural sciatica due to a vertebral osteochondroma (French), Neurochirurgie, 22 (1976), 301-306.

[5] J. M. Calhoun, W. M. Chadduck, and J. L. Smith, Single cervical exostosis. Report of a case and review of the literature, Surg Neurol, 37 (1992), 26-29.

[6] R. S. Cooke, W. J. Cumming, and R. A. Cowie, Osteochondroma of the cervical spine: case report and review of the literature, $\mathrm{Br}$ J Neurosurg, 8 (1994), 359-363.

[7] D. C. Dahlin and K. K. Unni, Bone Tumors: General Aspects and Data on 8,542 Cases, Charles C Thomas, Springfield, IL, 4th ed., 1986.

[8] E. Fiumara, T. Scarabino, G. Guglielmi, M. Bisceglia, and V. D'Angelo, Osteochondroma of the L-5 vertebra: a rare cause of sciatic pain. Case report, J Neurosurg, 91 (1999), 219-222.

[9] O. Gille, V. Pointillart, and J. M. Vital, Course of spinal solitary osteochondromas, Spine, 30 (2005), E13-E19.

[10] S. Govender and A. H. Parbhoo, Osteochondroma with compression of the spinal cord. A report of two cases, J Bone Joint Surg Br, 81 (1999), 667-669.

[11] D. Gürkanlar, A. Aciduman, A. Günaydin, H. Koçak, and N. Celik, Solitary intraspinal lumbar vertebral osteochondroma: a case report, J Clin Neurosci, 11 (2004), 911-913.

[12] I. H. Han and S. U. Kuh, Cervical osteochondroma presenting as brown-sequard syndrome in a child with hereditary multiple exostosis, J Korean Neurosurg Soc, 45 (2009), 309-311.

[13] E. T. Humbert, C. Mehlman, and A. H. Crawford, Two cases of osteochondroma recurrence after surgical resection, Am J Orthop, 30 (2001), 62-64.

[14] M. José Alcaraz Mexía, E. Izquierdo Núñez, C. Santonja Garriga, and R. María Salgado Salinas, Osteochondroma of the thoracic spine and scoliosis, Spine, 26 (2001), 1082-1085.

[15] A. Khosla, D. S. Martin, and E. E. Awwad, The solitary intraspinal vertebral osteochondroma. An unusual cause of compressive myelopathy: features and literature review, Spine, 24 (1999), 77-81.

[16] R. Louis, Chirurgie du Rachis: Anatomie Chirurgicale et Voies d'Abord, Springer-Verlag, Berlin, 1982.

[17] G. S. Novick, H. Pavlov, and P. G. Bullough, Osteochondroma of the cervical spine: report of two cases in preadolescent males, Skeletal Radiol, 8 (1982), 13-15.

[18] P. Roblot, M. Alcalay, F. Cazenave-Roblot, P. Levy, and D. Bontoux, Osteochondroma of the thoracic spine. Report of a case and review of the literature, Spine, 15 (1990), 240-243.

[19] K. Tajima, J. Nishida, K. Yamazaki, T. Shimamura, and M. Abe, Case report 545: Osteochondroma (osteocartilagenous exostosis) cervical spine with spinal cord compression, Skeletal Radiol, 18 (1989), 306-309.

[20] H. Y. Woo, D. C. Cho, K. J. Bae, and J. K. Sung, Solitary lumbar spinal osteochondroma presenting with sciatic pain: a case report, Korean J Spine, 7 (2010), 173-176.

[21] J. Xu, C. R. Xu, H. Wu, H. L. Pan, and J. Tian, Osteochondroma in the lumbar intraspinal canal causing nerve root compression, Orthopedics, 32 (2009), 133. 